\title{
The Predictive Factor for Favorable Outcome after Surgical Treatment of Benign Prostate Hyperplasia Performed by Young Urologist - Is Surgical Modality Important in Beginner Urologists?
}

Ki Hong Kim

Soon Chun Hyang University Cheonan Hospital

Si Hyun Kim

Soon Chun Hyang University Cheonan Hospital

Hee Jo Yang

Soon Chun Hyang University Cheonan Hospital

Doo Sang Kim

Soon Chun Hyang University Cheonan Hospital

Chang Ho Lee

Soon Chun Hyang University Cheonan Hospital

Youn Soo Jeon ( $\nabla$ ysurol@schmc.ac.kr)

Soon Chun Hyang University Cheonan Hospital

\section{Research Article}

Keywords: Endoscopes, Holmium Lasers, Prostatic hyperplasia, Transurethral resection of prostate, Urologists

Posted Date: December 31st, 2020

DOl: https://doi.org/10.21203/rs.3.rs-136711/v1

License: (c) (i) This work is licensed under a Creative Commons Attribution 4.0 International License. Read Full License

Version of Record: A version of this preprint was published at Soonchunhyang Medical Science on December 30th, 2021. See the published version at https://doi.org/10.15746/sms.21.014. 


\section{Abstract}

Background: To identify predictive factors for favorable outcomes after surgical treatments that were performed by beginner urologists in patients with benign prostate hyperplasia $(\mathrm{BPH})$, we retrospectively evaluated outcomes after holmium laser enucleation of the prostate (HoLEP) and transurethral resection of prostate (TURP) that were performed by two young urologists.

Methods: Of 80 patients who were treated with HoLEP or TURP, 31 (HoLEP) and 36 (TURP) patients who were followed up for 3 months were enrolled in this study. Preoperative and perioperative variables were evaluated to identify predictive factors for favorable outcome after surgical treatment for BPH.

Results: At 3 months postoperative after HoLEP or TURP, the median decrease in IPSS was 13.0. Patients whose IPSS decreased by over 13 points were categorized into a favorable response group after HoLEP or TURP. Univariate and multivariate logistic regression analyses were performed to identify predictors of favorable outcomes at 3 months after HoLEP or TURP, and the preoperative IPSS was identified as an independent predictor for favorable outcomes.

Conclusions: When young urologists plan to perform surgical treatment for BPH, they should consider that the severity of symptoms is the most important factor for favorable outcomes. The type of surgical modality for managing BPH is less important.

Trial registration : Soonchunhyang university institutional review board approval (No. 2017-07-022)

\section{Background}

Transurethral resection of the prostate (TURP) has been the gold standard for surgical treatment of lower urinary tract symptoms due to benign prostatic hyperplasia (BPH).[1] However, since Holmium laser enucleation of the prostate (HoLEP) was introduced as the treatment for benign prostatic hyperplasia $(\mathrm{BPH}),[2]$ several clinicians have suggested that HoLEP could replace TURP as the current gold standard for the treatment of BPH.[3]

While it has been reported that HoLEP is an admirable challenger of TURP, [4, 5] several clinicians hesitate to perform HoLEP because of its steep learning curve.[6-11] In addition, most young beginner urologists agonize over selecting HoLEP as a surgical modality for $\mathrm{BPH}$, because most previous reports on the learning curve associated with HoLEP included only expert surgeons. To the best of our knowledge, there is no comparative study in younger urologists regarding their learning curve for TURP and HoLEP. For this reason, the factors that should be considered for proper patient selection and surgical modality should be determined for young beginner urologists who decide to perform surgical treatment for BPH.

Authors have attempted to identify predictive factors for favorable outcome after HoLEP and TURP that were performed by young beginner urologists in $\mathrm{BPH}$, and tried to investigate whether surgical modality is an important factor. 


\section{Methods}

\section{Procedure}

Two young urologists performed surgical treatment for BPH after completing fellowship training for 2 years. HJY performed only HoLEP as the modality for surgical treatment of $\mathrm{BPH}$, and $\mathrm{KHK}$ performed only TURP in all cases.

A 72-W OmniPulse (Trimedyne Inc., Irvine, CA, USA) was used for the HoLEP, and the energy source consisted of a 72-W holmium: yttrium aluminum garnet laser with a 550- $\mu \mathrm{m}$ laser fiber. Enucleated prostatic tissue was removed using transurethral morcellation by use of a mechanical morcellator (Richard Wolf, Knittlingen, Germany) that was introduced through an offset rigid nephroscope. In cases of TURP, a standard Richard Wolf (Knittlingen, Germany) rectoscope and working elements were used, and a bipolar technique was applied in all TURP cases.

\section{Patients}

Forty consecutive patients who were treated with HoLEP by HJY and TURP by KHK for BPH were included in this study. HoLEP cases were performed between June 2014 and November 2016, and TURP cases were performed between April 2016 and March 2017. Of 80 patients who were treated with HoLEP or TURP, 31 (HoLEP) and 36 (TURP) patients who were followed up to 3 months were enrolled in this study. After receiving institutional review board approval (IRB no. 2017-07-022), we conducted a retrospective chart review of 31 (HoLEP) and 36 (TURP) patients.

\section{Clinical data and statistical analysis}

Age at time of surgery, body mass index (BMI), surgical modality for $\mathrm{BPH}$, medical history, preoperative prostate specific antigen (PSA), International Prostate Symptom Score (IPSS), quality of life (QoL) score, the maximum urinary flow rate (Qmax), post-void residual urine volume (PVR), total prostate volume, transition zone volume, and history of acute urinary retention (AUR) were estimated as preoperative variables for analysis, and weight of resected tissue, operative time, volume of resected tissue, resection speed, resected ratio, catheterization duration, and hospital days were used as perioperative variables. Resection speed was defined as the total amount of removed tissue after TURP or HoLEP in gm divided by total operative time in minutes,[12] and resected ratio was defined as volume of resected tissue divided by preoperative transition zone volume. Serum PSA for all included patients was assayed with a Cobas-e411 system (Roche Diagnostics), and prostate volume and transition-zone volume were estimated from transrectal ultrasound (TRUS) results using the prolate ellipsoid formula. IPSS, QoL score, and Qmax, which were evaluated at 3 months after surgical treatment, were estimated for postoperative outcomes.

The end point of this study was the degree of improvement in IPSS at 3 months after surgical treatment, and favor group after surgical treatment for BPH was defined as patients who experienced an improvement in IPSS over the median degree of improvement in IPSS. Baseline characteristics between 
HoLEP and TURP group were compared using an ANOVA for continuous variables and a Chi-square test for categorical variables, and to identify predictive factors for favorable outcome after surgical treatment for $\mathrm{BPH}$, univariate and multivariate logistic regression analysis were performed. Only the variables found to be significant on univariate analysis $(p<0.05)$ were entered into multivariate analysis. A receiver operating characteristic (ROC) curve was used to determine the optimal cut-off value of the significant predictive factor which was identified through logistic regression analysis. All statistical operations were performed using SPSS Statistics version 20.0.0 (IBM Corp., Armonk, NY, USA). A two-sided p-value of $<.05$ was considered statistically significant.

\section{Results}

The median age of all included patients was 75.50 years (range, 70.00-79.25), and the median degree of improvement in the IPSS was 13.00 (range, 6.00-19.00). The baseline characteristics of HoLEP and TURP group are presented in Table 1. HoLEP was significantly superior to TURP in terms of resected volume (gm) (median 32.0 vs. 16.0, $p=.013$ ), resected ratio (\%) (median 70.0 vs. 51.0, $p=.048$ ), duration of catheterization (days) (median 2.0 vs. 3.0, $p=.025$ ), and hospital day (median 2.0 vs. 3.0, $p=.029$ ). However, resection speed was not significantly different between the two groups $(p=.747)$. Operative time (min) was significantly shorter in TURP (median 115.0 vs. 70.0, $p<.001$ ). At 3 months after operation, there was no significant difference in urodynamic results between the two groups. Perioperative outcomes and postoperative outcomes at 3 months are presented in Table 2. 
Table 1

Patient characteristics

\begin{tabular}{|llll|}
\hline Preoperative Variables & HoLEP $(\mathrm{N}=31)$ & TURP $(\mathrm{N}=35)$ & P-value \\
\hline Age & $74.0(66.0-78.0)$ & $77.0(74.0-81.0)$ & .005 \\
\hline Past history & 6 & 6 & 1.000 \\
Cerebral vascular disease & 3 & 3 & 1.000 \\
Heart disease & 11 & 2 & .004 \\
Pulmonary disease & 10 & 10 & .793 \\
Diabetes & 17 & 25 & .204 \\
Hypertension & 2 & 3 & .558 \\
Chronic renal failure & & & .268 \\
\hline IPSS & $22.0(14.0-28.0)$ & $23.0(18.0-33.0)$ & .559 \\
\hline QoL score & $5.0(4.0-5.0)$ & $4.0(4.0-5.0)$ & .390 \\
\hline Max urine flow, $\mathrm{ml} / \mathrm{sec}$ & $5.8(4.1-8.6)$ & $6.8(4.6-9.6)$ & .283 \\
\hline PVR, ml & $139.0(78.3-250.3)$ & $116.0(62.5-170.0)$ & .111 \\
\hline Prostate size, $\mathrm{ml}$ & $64.0(53.0-99.0)$ & $58.0(40.0-81.0)$ & .070 \\
\hline Transition zone size, $\mathrm{ml}$ & $39.0(30.0-60.0)$ & $24.0(17.0-50.0)$ & .818 \\
\hline PSA, ng/ml & $4.2(1.9-8.0)$ & $4.1(2.0-7.2)$ & .804 \\
\hline Urinary retention & 14 & 14 & $24.5(22.6-26.8)$ \\
\hline Body mass index, kg/m ${ }^{2}$ & $23.9(22.2-26.3)$ & & .958 \\
\hline
\end{tabular}


Table 2

Perioperative outcomes and postoperative outcomes at 3months

\begin{tabular}{|llll|}
\hline Perioperative Variables & HoLEP $(\mathrm{N}=31)$ & TURP $(\mathrm{N}=35)$ & P-value \\
\hline Operative time, min & $115.0(80.0-165.0)$ & $70.0(50.0-95.0)$ & $<.001$ \\
\hline Resection speed, gm/min & $0.24(0.17-0.32)$ & $0.24(0.15-0.34)$ & .747 \\
\hline Resected tissue, gm & $32.0(12.0-45.0)$ & $16.0(7.5-30.0)$ & .013 \\
\hline Resected ratio, \% & $70.0(45.0-82.0)$ & $51.0(38.0-68.0)$ & .048 \\
\hline Catheterization duration & $2.0(2.0-2.0)$ & $3.0(2.0-3.0)$ & .025 \\
\hline Hospital day & $2.0(2.0-2.0)$ & $3.0(2.0-4.0)$ & .029 \\
\hline Postoperative Outcomes at 3months & & & .562 \\
\hline IPSS & $11.0(6.0-16.5)$ & $9.5(6.0-14.0)$ & .462 \\
\hline QoL score & $3.0(1.5-4.0)$ & $2.5(1.0-3.0)$ & .782 \\
\hline Max urine flow, ml/sec & $14.3(11.1-23.7)$ & $16.5(11.4-21.4)$ & .121 \\
\hline PVR, ml & $40.0(15.0-82.0)$ & $71.0(34.0-89.0)$ &. \\
\hline
\end{tabular}

After the favorable group was defined as patients who experienced an improvement of over 13 in the IPSS, univariate and multivariate logistic regression analyses were performed to identify the predictive factors for being included in the favorable group after surgical treatment for BPH (Table 3 ). These analyses identified preoperative IPSS as an independent predictor of favorable outcome $(O R=1.307, P$ $<$.001). Surgical modality was not a significant predictor. 
Table 3

Predictors of favorable outcome after surgical treatment for benign prostate hyperplasia

\begin{tabular}{|lll|}
\hline Univariate Analysis & & \\
\hline Variables & OR & P-value \\
\hline Modality & $1.205(0.447-3.250)$ & .712 \\
\hline Age & $0.995(0.924-1.071)$ & .890 \\
\hline Past history & $0.635(0.178-2.267)$ & .484 \\
Cerebral vascular disease & $0.966(0.180-5.193)$ & .967 \\
\hline Heart disease & $0.635(0.178-2.267)$ & .484 \\
\hline Pulmonary disease & $0.822(0.280-2.414)$ & .721 \\
\hline Diabetes & $1.206(0.432-3.367)$ & .721 \\
\hline Hypertension & $0.301(0.030-3.064)$ & .311 \\
Chronic renal failure & $0.831(0.302-2.282)$ & .719 \\
\hline Urinary retention & & .979 \\
\hline IPSS & $1.294(1.145-1.463)$ & $<.001$ \\
\hline QoL score & $2.302(1.334-3.974)$ & .003 \\
\hline Max urine flow, ml/sec & $0.920(0.813-1.093)$ & .180 \\
\hline PVR, ml & $1.002(0.998-1.005)$ & .366 \\
\hline Prostate size, ml & $0.993(0.978-1.008)$ & .363 \\
\hline Transition zone size, $\mathrm{ml}$ & $0.991(0.972-1.012)$ & .399 \\
\hline PSA, ng/ml & $1.097(0.979-1.229)$ & .112 \\
\hline Body mass index, kg/m² & $0.889(0.742-1.066)$ & .204 \\
\hline Operative time, min & $1.000(0.991-1.009)$ & .958 \\
\hline Retrieval rate, gm/min & $3.930(0.042-366.152)$ & .554 \\
\hline Resected tissue, gm & $1.005(0.978-1.034)$ & .698 \\
\hline Enucleation ratio, \% & $2.727(0.537-13.846)$ & .226 \\
\hline Catheterization duration & $0.877(0.682-1.128)$ & .877 \\
\hline Hospital day & $1.010(0.823-1.239)$ & .925 \\
\hline Multivariate analysis & & \\
\hline
\end{tabular}




\begin{tabular}{|lll|}
\hline Univariate Analysis & & \\
\hline Variables & OR & P-value \\
\hline IPSS & $1.307(1.138-1.501)$ & $<.001$ \\
\hline QoL score & $0.889(0.412-1.919)$ & .764 \\
\hline
\end{tabular}

In ROC curve, preoperative IPSS 20.5 was identified as the optimal cut-off value for favorable postoperative outcome and the areas under the ROC curves (AUC) was 0.887 (Fig. 1). Sensitivity and specificity were $93.8 \%$ and $67.7 \%$ in preoperative IPSS 20.5 , which was identified as the optimal cut-off value, respectively.

\section{Discussion}

Several authors have reported comparison studies between TURP and HoLEP.[13-21] Gilling et al. reported outcomes at 1,2, and 7 years after HoLEP or TURP.[13-15] They demonstrated that there was no difference in postoperative urodynamic results between TURP and HOLEP. However, they reported that HoLEP was superior to TURP in duration of catheterization and the duration of hospital stay. The results of several prospective and randomized trials are reported.[17-20] Authors of prospective and randomized trials have reported that HoLEP is superior to TURP in duration of catheterization and hospital stays. In addition, they have demonstrated that TURP is superior to HoLEP in operative time. Several authors have suggested that there was no difference in postoperative urodynamic results between HoLEP and TURP, $[17,19]$ while others argue that HoLEP is slightly superior to TURP in this aspect.[18, 20] Procedures in previous reports about the comparison between TURP and HoLEP were performed mostly by experts who have extensive experience of transurethral surgery. To the best of our knowledge, there is no comparative study of learning curves between TURP and HoLEP. For this reason, the predictive factor that is useful for young beginner urologists who agonize about selecting a surgical modality before performing surgical treatment for BPH should be identified.

Preoperative IPSS has been known as one of the predictors for favorable outcome in BPH patients who were treated using TURP.[22-24] Hakenberg et al. and Chang et al. reported that patients showing severe preoperative IPSS obtained a greater improvement in postoperative IPSS than patients showing mild preoperative IPSS. A preoperative IPSS value of 17 is the cut-off value for the prediction of favorable postoperative outcome.[23, 24] However, preoperative IPSS was not an independent predictor of favorable outcomes after TURP in these studies because they did not perform multivariate analysis. Moreover, these studies had a limitation in that they arbitrarily defined significant symptomatic postoperative improvement as a decrease of 10 or more symptom score points. Bruskewitz et al. also reported that preoperative IPSS could be one of the predictors for favorable outcome after TURP.[22] However, they also performed a multivariate analysis.

The authors identified that preoperative IPSS was a statistically significant independent predictor for favorable outcomes after surgical treatment in BPH unlike in previous reports, and that surgical modality 
is not an important factor for the improvement of lower urinary tract symptoms after surgical treatment in $\mathrm{BPH}$. Especially, this report can be useful for beginner urologists who do not overcome the learning curve of surgical treatment for $\mathrm{BPH}$, because this study was based on the experiences of young beginner urologists.

HoLEP is known as having a steep learning curve, and several studies have reported on this subject.[611] By comparing outcomes in HoLEP that were performed by an unexperienced urologist and by a urologist with extensive experience with transurethral surgery, El-Hakim et al. suggested that extensive experience with transurethral surgery is a prerequisite for the success of HoLEP.[8] Shah et al. reported that an urologist inexperienced with HoLEP could perform the procedure with reasonable efficiency after 50 cases; their prospective study was based on the experiences of a surgeon who has performed transurethral surgery in 150 cases.[11] Bae et al. also reported that they reached a stable enucleation and a morcellation efficiency state after 30 cases and 20 cases, respectively.[6] Meanwhile, Brunckhorst et al. reported that they experienced a learning curve of 40-60 cases for the HoLEP procedure in their retrospective study, which was based on the experiences of a surgeon who performed transurethral surgery in 500 cases.[7] A recently published report demonstrated that 20 cases are probably sufficient to give the surgeon the impetus to continue with the technique,[10] and Elshal et al. suggested that preoperative prostate volume, the number of previously performed cases, and case density are the main influential factors in the learning curve.[9]

Nevertheless, the authors indicate that young beginner urologists should concentrate their attention on the severity of symptoms rather than on the selection of surgical modality when they consider surgical treatment, such as HoLEP or TURP, for patients who have lower urinary tract symptoms. In addition, since the preoperative IPSS is more of an independent predictor than the surgical modality for improvement after surgical treatment for $\mathrm{BPH}$, the authors recommend that it is unnecessary to hesitate to perform HoLEP just due to its steep learning curve.

This report has the limitation that this study was designed retrospectively and the number of included patients was relatively small. However, the authors judge that the scale of this study, which was performed in 40 patients who were treated with HoLEP or TURP is sufficient for evaluating initial experience because a learning curve of 20-60 cases was shown to be necessary for learning the HoLEP procedure through previously published reports.

The results that are reported in the current study need to be confirmed and validated by analyzing data from a prospective study.

\section{Conclusions}

When young and beginner urologists plan to perform surgical treatment for BPH, they should consider that the severity of symptom is the most important factor for favorable outcomes. Since the type of surgical modality for managing $\mathrm{BPH}$ is not an important factor, the authors suggest to young beginner urologists that it is unnecessary to hesitate to perform HoLEP due to its steep learning curve. 


\section{Abbreviations}

$\mathrm{BPH}$ : Benign prostate hyperplasia

HoLEP : Holmium laser enucleation of the prostate

TURP : Transurethral resection of prostate

$\mathrm{BMI}$ : Body mass index

PSA : Prostate specific antigen

IPSS : International Prostate Symptom Score

QoL : Quality of life

Qmax : The maximum urinary flow rate

PVR : Post-void residual urine volume

AUR : Acute urinary retention

TRUS : Transrectal ultrasound

ROC : Receiver operating characteristic

AUC : The areas under the ROC curves

\section{Declarations}

\section{Ethics approval and consent to participate}

This study was performed at the Department of Urology, Soonchunhyang University Cheonan Hospital, Republic of Korea. Soonchunhyang university institutional review board approved this study (No. 201707-022). All patients were provided written informed consent and agreed that the data will be used for this retrospective study. All methods were carried out in accordance with relevant guidelines and regulations.

\section{Consent for publication}

Not applicable.

\section{Availability of data and materials}


The datasets used and/or analysed during the current study available from the corresponding author on reasonable request.

\section{Competing interests}

The authors declare that they have no conflicts of interest.

\section{Funding}

This work was supported by the "Soonchunhyang University Research Fund"

\section{Authors' contributions}

Ki Hong Kim: Conceptualization, Data curation, Methodology, Validation, Investigation, Writing-original draft.

Si Hyun Kim, Hee Jo Yang, Doo Sang Kim, Chang Ho Lee: Data curation, Validation, Investigation

Youn Soo Jeon: Conceptualization, Data curation, Methodology, Validation, Investigation, Writing-original review \& editing.

All authors read and approved the final manuscript.

\section{Acknowledgements}

This manuscript was prepared with the assistance of EDITAGE, an English-language scientific editing company.

\section{References}

1. Rassweiler J, Teber D, Kuntz R, Hofmann R: Complications of transurethral resection of the prostate (TURP)-incidence, management, and prevention. European urology 2006, 50(5):969-979; discussion 980.

2. Gilling PJ, Cass CB, Malcolm AR, Fraundorfer MR: Combination holmium and Nd:YAG laser ablation of the prostate: initial clinical experience. Journal of endourology / Endourological Society 1995, 9(2):151-153.

3. van Rij S, Gilling PJ: In 2013, holmium laser enucleation of the prostate (HoLEP) may be the new 'gold standard'. Current urology reports 2012, 13(6):427-432. 
4. Cornu JN, Ahyai S, Bachmann A, de la Rosette J, Gilling P, Gratzke C, McVary K, Novara G, Woo H, Madersbacher S: A Systematic Review and Meta-analysis of Functional Outcomes and Complications Following Transurethral Procedures for Lower Urinary Tract Symptoms Resulting from Benign Prostatic Obstruction: An Update. European urology 2015, 67(6):1066-1096.

5. Oelke M, Bachmann A, Descazeaud A, Emberton M, Gravas S, Michel MC, N'Dow J, Nordling J, de la Rosette $\mathrm{JJ}$ : EAU guidelines on the treatment and follow-up of non-neurogenic male lower urinary tract symptoms including benign prostatic obstruction. European urology 2013, 64(1):118-140.

6. Bae J, Oh SJ, Paick JS: The learning curve for holmium laser enucleation of the prostate: a singlecenter experience. Korean journal of urology 2010, 51(10):688-693.

7. Brunckhorst O, Ahmed K, Nehikhare O, Marra G, Challacombe B, Popert R: Evaluation of the Learning Curve for Holmium Laser Enucleation of the Prostate Using Multiple Outcome Measures. Urology 2015, 86(4):824-829.

8. El-Hakim A, Elhilali MM: Holmium laser enucleation of the prostate can be taught: the first learning experience. BJU international 2002, 90(9):863-869.

9. Elshal AM, Nabeeh H, Eldemerdash Y, Mekkawy R, Laymon M, El-Assmy A, El-Nahas AR: Prospective Assessment of Learning Curve of Holmium Laser Enucleation of the Prostate for Treatment of Benign Prostatic Hyperplasia Using a Multidimensional Approach. The Journal of urology 2016.

10. Robert G, Cornu JN, Fourmarier M, Saussine C, Descazeaud A, Azzouzi AR, Vicaut E, Lukacs B: Multicentre prospective evaluation of the learning curve of holmium laser enucleation of the prostate (HoLEP). BJU international 2016, 117(3):495-499.

11. Shah HN, Mahajan AP, Sodha HS, Hegde S, Mohile PD, Bansal MB: Prospective evaluation of the learning curve for holmium laser enucleation of the prostate. The Journal of urology 2007, 177(4):1468-1474.

12. Ahyai SA, Chun FK, Lehrich K, Dahlem R, Zacharias MS, Fisch MM, Kuntz RM: Transurethral holmium laser enucleation versus transurethral resection of the prostate and simple open prostatectomywhich procedure is faster? The Journal of urology 2012, 187(5):1608-1613.

13. Gilling PJ, Kennett KM, Fraundorfer MR: Holmium laser resection v transurethral resection of the prostate: results of a randomized trial with 2 years of follow-up. Journal of endourology / Endourological Society 2000, 14(9):757-760.

14. Gilling PJ, Mackey M, Cresswell M, Kennett K, Kabalin JN, Fraundorfer MR: Holmium laser versus transurethral resection of the prostate: a randomized prospective trial with 1-year followup. The Journal of urology 1999, 162(5):1640-1644.

15. Gilling PJ, Wilson LC, King CJ, Westenberg AM, Frampton CM, Fraundorfer MR: Long-term results of a randomized trial comparing holmium laser enucleation of the prostate and transurethral resection of the prostate: results at 7 years. BJU international 2012, 109(3):408-411.

16. Gupta N, Sivaramakrishna, Kumar R, Dogra PN, Seth A: Comparison of standard transurethral resection, transurethral vapour resection and holmium laser enucleation of the prostate for managing benign prostatic hyperplasia of $>\mathbf{4 0}$ g. BJU internationa/2006, 97(1):85-89. 
17. Montorsi F, Naspro R, Salonia A, Suardi N, Briganti A, Zanoni M, Valenti S, Vavassori I, Rigatti P: Holmium laser enucleation versus transurethral resection of the prostate: results from a 2-center prospective randomized trial in patients with obstructive benign prostatic hyperplasia. The Journal of urology 2008, 179(5 Suppl):S87-90.

18. Tan AH, Gilling PJ, Kennett KM, Frampton C, Westenberg AM, Fraundorfer MR: A randomized trial comparing holmium laser enucleation of the prostate with transurethral resection of the prostate for the treatment of bladder outlet obstruction secondary to benign prostatic hyperplasia in large glands (40 to 200 grams). The Journal of urology 2003, 170(4 Pt 1):1270-1274.

19. Westenberg A, Gilling P, Kennett K, Frampton C, Fraundorfer M: Holmium laser resection of the prostate versus transurethral resection of the prostate: results of a randomized trial with 4-year minimum long-term followup. The Journal of urology 2004, 172(2):616-619.

20. Wilson LC, Gilling PJ, Williams A, Kennett KM, Frampton CM, Westenberg AM, Fraundorfer MR: A randomised trial comparing holmium laser enucleation versus transurethral resection in the treatment of prostates larger than $\mathbf{4 0}$ grams: results at 2 years. European urology 2006, 50(3):569573.

21. Yin L, Teng J, Huang CJ, Zhang X, Xu D: Holmium laser enucleation of the prostate versus transurethral resection of the prostate: a systematic review and meta-analysis of randomized controlled trials. Journal of endourology / Endourological Society 2013, 27(5):604-611.

22. Bruskewitz RC, Reda DJ, Wasson JH, Barrett L, Phelan M: Testing to predict outcome after transurethral resection of the prostate. The Journal of urology 1997, 157(4):1304-1308.

23. Chuang FP, Lee SS, Wu ST, Yu DS, Chen HI, Chang SY, Sun GH: Change in International Prostate Symptom Score after transurethral prostatectomy in Taiwanese men with benign prostate hyperplasia: use of these changes to predict the outcome. Archives of andrology 2003, 49(2):129137.

24. Hakenberg OW, Pinnock CB, Marshall VR: Does evaluation with the International Prostate Symptom Score predict the outcome of transurethral resection of the prostate? The Journal of urology 1997 , 158(1):94-99.

\section{Figures}




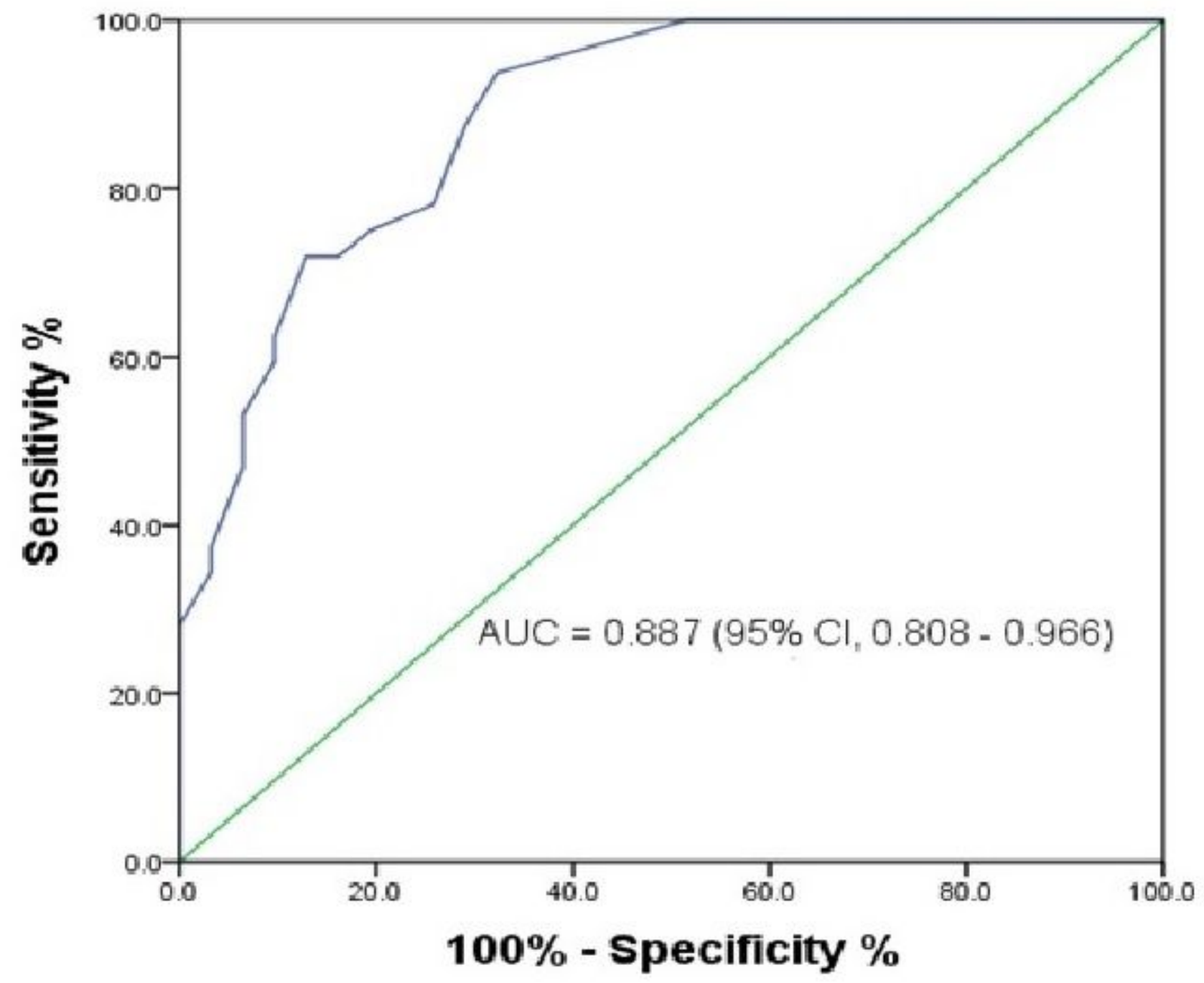

Figure 1

Receiver operating characteristic curve for identifying the optimal cut-off value for favorable postoperative outcome after surgical treatment for benign prostate hyperplasia. 